\title{
Une approche des raisonnements émergents de l'interlocution : la logique interlocutoire
}

Alain Trognon, Martine Batt, Manuel Rebuschi et Christine Sorsana

\section{(2) OpenEdition}

\section{Journals}

\section{Édition électronique}

URL : http://journals.openedition.org/pratiques/1632

DOI : $10.4000 /$ pratiques. 1632

ISSN : 2425-2042

\section{Éditeur}

Centre de recherche sur les médiations (CREM)

\section{Édition imprimée}

Date de publication : 15 décembre 2010

Pagination : 131-154

\section{Référence électronique}

Alain Trognon, Martine Batt, Manuel Rebuschi et Christine Sorsana, « Une approche des raisonnements émergents de l'interlocution : la logique interlocutoire », Pratiques [En ligne], 147-148 | 2010, mis en ligne le 15 juin 2010, consulté le 01 mai 2019. URL : http:// journals.openedition.org/pratiques/1632 ; DOI : 10.4000/pratiques.1632 


\title{
Une approche des raisonnements émergents de l'interlocution : la logique interlocutoire
}

\author{
Alain Trognon, Martine Batt, \\ Manuel Rebuschi, Christine Sorsana \\ Université Nancy 2
}

\section{Introduction}

Toutes les espèces animales vivant en société ont l'interaction directe pour milieu naturel. L'espèce humaine ne fait pas exception sauf que pour elle l'interaction s'enrichissant du talk, c'est le talk-in-interaction, selon la belle expression de Schegloff (1991), qui constitue son milieu naturel.

Aujourd'hui (Trognon \& Batt, 2010 ; Trognon \& Bromberg, 2007), cette conclusion n'est plus à proprement parler une thèse mais un truisme. Les neurosciences nous ont en effet récemment appris que l'interaction n'était pas seulement « autour» de nous, mais aussi bien « en » nous, réfléchie dans l'organisation de notre cerveau. Et la psychologie du développement nous enseigne que le nourrisson s'engage très précocement avec ceux qui prennent soin de lui dans des proto-conversations présentant déjà la propriété de séquentialité qui est à la base de toute conversation (Trognon \& Sorsana, 2005). On serait donc fondé à conclure avec Garfinkel (1990 : 26-27) que : " every topic of logic, order, reason, meaning, and method is to be discovered and is discoverable, and is respecified and respecifiable only as locally produced, naturally accountable phenomena of order. The phenomena are immortal, ordinary society's commonplace, vulgar, familiar, unavoidable, irremediable and uninteresting "work of the street" ». Par conséquent, d'un point de vue plus psychologique, on devrait affirmer que : « le sujet cognitif n'est pas une monade : [qu'] il interagit en permanence avec son environnement, et en particulier avec d'autres sujets [...] et [que] cette interaction met en jeu de façon prioritaire, le langage [...: :] rendre compte de cette interaction, et notamment de l'interaction verbale, qui en est sans doute la forme la plus élaborée, constitue un défi essentiel pour la psychologie cognitive [..., q qu'elle doit relever » (Caron, 1997 : 233). Aussi les chercheurs en psychologie conséquents devraient-ils s'empresser d'imaginer des 
dispositifs d'observation et d'analyse des «facultés » psychologiques faisant fond sur leur contexte naturel d'accomplissement de façon à constituer une « psychologie concrète », c'est à dire, comme aurait dit Politzer, une psychologie véritable de la mémoire, du langage, du raisonnement, de l'affectivité, etc.

La psychologie sociale génétique a incontestablement relevé ce défi en matière de production des données en inventant un schéma d'expérimentation propre à éprouver la conjecture que les individus améliorent leurs "facultés » quand ils les exercent dans l'interaction. Grâce à ce schéma d'expérimentation [Pré-test / (Groupe contrôle en situation individuelle vs Groupe expérimental en situation d'interaction) / Post-test immédiat et différé], devenu depuis incontournable dans ce genre de recherches, preuve a été faite que l'interaction est un accélérateur incontestable des acquisitions à travers divers processus dont le plus connu est le conflit socio-cognitif (Perret-Clermont, 1979 ; Sorsana, 1999). Cependant, des progrès semblables n'ont pas été accomplis en matière d'analyse des données, car comme l'écrivent Wertsch et Sammarco (1988:411) le fait de posséder de nombreuses données prouvant l'influence bénéfique de l'interaction dans l'acquisition « ne résout pas encore le problème des mécanismes qui font la transition entre le fonctionnement interpsychologique et le fonctionnement intrapsychologique $»$.

Pour résoudre ce problème, il semble que deux conditions doivent être satisfaites. Il faut tout d'abord enrichir les données expérimentales en associant aux performances des dyades du groupe expérimental les interrelations, par exemple les discussions, que ces performances ont conclues, c'est-à-dire en associant aux performances leurs microgenèses. Ce qui n'était pas encore possible avant la dernière guerre mondiale est devenu parfaitement abordable de nos jours et on peut s'étonner que l'enregistrement et l'analyse des données expérimentales produites en situation d'interaction ne soient pas devenu une pratique courante dans les expérimentations traitant des contextes interactifs. Les performances sont en effet des données trop grossières pour approcher toute la finesse des mécanismes dont elles constituent l'aboutissement. «Dans la notion de microgenèse, se trouve l'idée de travailler à une autre échelle temporelle que celle de la macrogenèse, mais surtout d'analyser les conduites cognitives dans le plus grand détail et dans toute leur complexité naturelle. L'étude des microgenèses met en évidence les caractéristiques du processus interactif entre le sujet [, son partenaire] et l'objet qui avait été analysé de façon trop globale par Piaget. Elle permet de déceler la coordination et l'intégration éventuelles des solutions et modèles partiels successifs du sujet [et de son partenaire] » (Inhelder \& de Caprona, 1992, p. 24, c'est nous qui avons introduit les crochets et leur contenu). Il faut deuxièmement concevoir un langage formalisant dans le détail l'accomplissement du raisonnement dans l'interlocution.

Le but que nous poursuivons principalement dans cet article est d'exposer un tel langage, non pas abstraitement mais en en illustrant l'emploi pour explorer la microgenèse de la découverte de la solution d'un problème arithmétique par deux garçons discutant à cette fin. Nous travaillerons sur la base d'un corpus provenant de l'enregistrement d'une dyade appartenant au groupe expérimental d'une expérimentation montrant que les dyades sont plus performantes que les sujets isolés dans la résolution de ce problème. En examinant cette situation de 
résolution interactive ou dialoguée d'un problème, nous montrerons que l'emploi de ce langage permet d'expliciter dans son ensemble le processus d'interaction ayant conduit à la découverte de la solution, d'identifier ses composants nodaux, surtout ceux qui prouvent la rationalité et plus exactement la validité logique de l'interaction de résolution, enfin de circonscrire quelques propriétés psycho-socio-discursives formant la base du processus interactif en cause.

Nous commencerons par présenter l'expérimentation dont est issu l'enregistrement que nous examinerons ensuite. La démarche mise en œuvre pour analyser l'enregistrement sera abordée dans un second temps. Nous en exposerons les buts, les contraintes qui lui sont imposées et le langage qui en découle. Nous consacrerons la troisième partie de notre travail à l'analyse de la microgenèse de la résolution dialoguée du problème qui a été posé aux enfants.

\section{Présentation de l'extrait analysé}

L'enregistrement que nous allons étudier provient d'un travail expérimental conduit par Säljö et Wyndahamn (1990) publié dans le British Journal of Educational Psychology. Dans cette expérimentation il était demandé à des enfants de 9-10 ans de résoudre des problèmes arithmétiques d'abord isolément puis en dyades. Dans ces deux situations successives, les cinq problèmes qui étaient proposés aux enfants leur étaient présentés comme des problèmes de multiplication alors qu'un d'entre eux dans la première situation et deux d'entre eux dans la seconde requerraient pour leur résolution une multiplication et une division. Dans les deux situations le contrat de communication didactique était donc enfreint. Le but de l'expérimentation était d'établir que la collaboration des enfants facilite leur découverte de la solution tout en les libérant de leur dépendance vis-à-vis d'un contrat de communication didactique défectueux.

Jean et Marc étaient les deux enfants composant la dyade ayant produit le discours rapporté ici. Ils avaient dix ans chacun, avaient reçu un enseignement sur la division dans le cadre de leur programme scolaire et résolvaient l'un des deux problèmes intitulé «multiplication » mais dont la solution demandait selon les expérimentateurs au moins une division. Voici l'énoncé du problème tel qu'il avait été donné aux enfants :

Un commerçant a 672 cassettes vidéo à ranger. il peut en ranger 32 par étagère. Sachant qu'une bibliothèque comporte 7 étagères, de combien de bibliothèques $a-t$-il besoin pour ranger ces cassettes?

Les initiales J (Jean) et M (Marc) désignent les enfants. E désigne l'expérimentatrice. Les tours de parole sont notés successivement. Certains tours de parole comportant plusieurs énoncés, nous les décomposerons autant que de besoin par les lettres de l'alphabet, par exemple $34 \mathrm{~J}$ sera décomposé en $34 \mathrm{Ja}, 34 \mathrm{Jb}$, $34 \mathrm{jc}, 34 \mathrm{Jd}, 34 \mathrm{Je}$. Les observations de l'expérimentatrice sont portées entre crochets.

$[\ldots]$

25 M: [il lit l'énoncé] "combien y a-t-il"

$26 \mathrm{~J}$ : "d'étagères" "a-t-ill besoin d'étagères pour ranger ces cassettes ?" 
$27 M$ : d'étagères quoi de bibliothèques il y en a 7 on peut en mettre 32 dans une

$28 \mathrm{~J}$ : ilya 7 étages

29 M: pis $32 \ldots 32$ ouais ben il faut faire...

$30 \mathrm{~J}$ : faut diviser par 32 ? [il le regarde]

$31 \mathrm{M}$ : ou peut-être fois aussi... parce que 32 fois "combien"

$32 \mathrm{~J}$ : à moins qu'on arrive chaque fois à 32

$33 \mathrm{M}$ : attends

$34 \mathrm{~J}$ : 7 fois $32 . . .14$ [il calcule] $m m h$ !... possible par étagère tu peux en mettre $200 . . .224$ alors il y a qu'à enlever à chaque fois 224

$35 \mathrm{M}$ : par étagère... 32 Ah ouais 224 ... ben 600

$36 \mathrm{~J}$ : 224 ouais ! par BIBLIOTHEQUE [accentué]

$37 \mathrm{M}$ : ah oui ben fais sur le brouillon

$38 \mathrm{~J}$ : ouais

39 M: 672 moins 224 moins 224 moins 224 (...)

$40 \mathrm{~J}$ : [à voix basse] $672 \ldots$ moins 224

$41 \mathrm{M}$ : faut prendre

$42 \mathrm{~J}:$ là!

$43 \mathrm{M}$ : faut prendre faut prendre 6 [soupir] c'est pas la joie

$44 \mathrm{~J}$ : quatre voilà ! puis là tu peux encore en mettre deux parce que c'est 224 là tu peux en mettre encore deux alors ça veut dire qu'il en a besoin de trois

$45 \mathrm{M}$ : mais si... mais...

$46 \mathrm{~J}$ : oui!?

$47 M$ : essaie d'en mettre deux... tu mets... ben fais comme ça... attends je le fais... je

$48 \mathrm{~J}$ : là on en a déjà enlevé une

49 M: 224

$50 \mathrm{~J}: 224$ ouais

$51 \mathrm{M}$ : ben on en a besoin de trois moins 224

$52 \mathrm{~J}$ : alors on fait comme ça... tu mets 672

$53 \mathrm{M}$ : ouais!

$54 \mathrm{~J}: 72$ moins 224 moins 224 moins 224 égal... euh là là ça fait 0 après [chuchote] 224 est égal... tu veux pas mettre des espaces?

$55 \mathrm{M}$ : euh! encore une fois il ya

$56 \mathrm{~J}$ : puis après ça fait $0 . .$. alors euh 3 étagères... euh 3 ... bibliothèques

$57 \mathrm{M}$ : [chuchote] euh! qu'est-ce qu'il faut marquer pour marquer trois étagères?

$58 \mathrm{~J}$ : ben ... on met... il faut 3 étagères!

$59 \mathrm{M}: 3 ! ?$

$60 \mathrm{~J}: 3$ !

$61 \mathrm{M}$ : 3 bibliothèques

$62 \mathrm{~J}$ : 3 bibliothèques! ben 3 bibliothèques on doit mettre parce que ça fait

$63 \mathrm{M}$ : bi-bli-o-thèque [il écrit en faisant une faute que J relève immédiatement après]

$64 \mathrm{~J}$ : biliothèque?

$65 \mathrm{M}$ : bibliothèque [rectifie et accentue]

$66 \mathrm{~J}$ : tu as oublié le L

$67 \mathrm{M}$ : bibli

$69 \mathrm{~J}$ : bibliothèque non il est icile $L$

70 M: ah! ouais j'ai oublié le $B$ [J et M sourient] 
$71 \mathrm{~J}$ : siyaun Letya pas de $B$

$72 M$ : [chuchote]... thèque voilà !

[les enfants ont écrit au propre 672-224-224-224=0

3 bibliothèques]

\section{Notions de logique interlocutoire}

Le but de la logique interlocutoire est d'expliciter formellement les événements indissolublement psycho-socio-discursifs qui surviennent « naturellement » dans le talk-in-interaction ou interlocution (Trognon et Batt, 2010). Quatre propriétés génériques déterminent le mode d'existence empirique de ces événements. La logique interlocutoire associe à ces propriétés un langage (le langage de la sémantique générale, dont (en première approche) le langage de la logique modale des prédicats du premier ordre) et des méthodes logiques (déduction naturelle, calcul des séquents, logiques dialogiques) dont la combinaison est censée formaliser adéquatement les propriétés génériques des événements interlocutoires. Nous allons maintenant exposer aussi intuitivement que possible cette démarche en empruntant nos illustrations au corpus ci-dessus.

\subsection{Première propriété : les événements interlocutoires sont à la fois actionnels et représentationnels}

Comme les objets empiriques qui assurent la double fonction actionnelle et représentationnelle de la parole-dans-l'interaction sont au départ les « actes de langage » élémentaires et ensuite leurs différentes combinaisons en objets d'ordre supérieur, la syntaxe de la logique interlocutoire doit au moins comporter des symboles de ces éléments de départ. Cela explique pourquoi la "sémantique générale », logique élaborée progressivement par Searle et Vanderveken à partir de la découverte des actes de langage par Austin assure le soubassement de la logique interlocutoire. Les actes de langage de la sémantique générale sont en effet les actes de langage qui sont directement « codés » ${ }^{(1)}$ dans les énoncés d'une langue naturelle par sa grammaire. Constituant les arguments de départ sur lesquels « travaillent» les processus (ou fonctions) de reconnaissance et d'inférence grâce auxquelles les interlocuteurs parviennent à individualiser (sens du locuteur) puis à collectiviser (sens des interlocuteurs) le sens assumé par les interlocuteurs (Trognon, 2002; Trognon \& Batt, 2010), ils sont les pièces ultimes des structures complexes innombrables qui se forment dans l'interlocution, des échanges élémentaires (par exemple les paires adjacentes de l'Analyse Conversationnelle comme la paire question-réponse (Carlson,1983) aux jeux de dialogues (Trognon, Batt, Sorsana et alii, 2010 ; Trognon \& Batt, 2010 ; Trognon \& Bromberg, 2008; Walton, 2007) simples ou complexes.

Voyez par exemple comment s'élabore progressivement et collaborativement dans $(62 \mathrm{~J}, 63 \mathrm{M}, 64 \mathrm{~J})$ la représentation de l'acte de langage accomplit par Jean en 62J. ben 3 bibliothèques on doit mettre décrit la tâche qui s'impose aux en-

(1) «Directement» ne veut pas dire « exactement ». Le codage des actes illocutoires dans les langues naturelles n'est pas exempt d'ambiguité. De plus, les actes de langage littéralement exprimés dans les énoncés ne sont pas nécessairement les actes de langage effectifs de l'interlocution (Trognon, 2002 ; Trognon \& Batt, 2010). 
fants à ce moment de leur réflexion. L'énoncé se présente littéralement comme une réponse et donc une assertion. Mais on voit bien que cette assertion est prise par Marc au tour de parole suivant comme une proposition puisqu'il en réalise le contenu propositionnel et que cette action est ratifiée par Jean lorsqu'il demande indirectement (au moyen d'une question) à Marc de corriger son travail. Par conséquent, tandis que leur travail progresse, les enfants s'accordent parallèlement sur l'acte de langage que Jean est censé avoir accompli en énonçant 62J (Trognon, 2002 ; Trognon et Batt, 2010).

Nous analyserons donc la plupart des énoncés produits en contexte par la forme $\mathrm{F}(\mathrm{P})$, dans laquelle $\mathrm{F}$ représente la fonction actionnelle, la force, de l'acte de langage et $\mathrm{P}$ représente sa fonction représentationnelle, son contenu propositionnel, ce dernier exprimé grâce au langage de la logique des prédicats du premier ordre modalisée et à des langages logiques plus « primitifs ». C'est en confrontant cette représentation littérale dans l'énoncé d'un acte de langage potentiellement accompli par le locuteur avec des connaissances relatives aux contextes d'émission de l'énoncé que nous approcherons l'acte de langage (indirect ou implicite) qu'il a peut-être effectivement accompli dans l'interlocution (Trognon et Coulon, 2001).

\subsection{Deuxième et troisième propriétés : séquentialité et architecturalité des événements interlocutoires}

Les événements interlocutoires s'accomplissent séquentiellement, à la suite les uns des autres, en se présentant comme des concaténations. Parallèlement, ils s'organisent hiérarchiquement (plus ou moins librement) en différentes architectures (plus ou moins régulières). Cette apparence de linéarité est une propriété bien connue de la phrase, du moins depuis les débuts de la grammaire générative. Elle est élargie ici à l'interlocution.

$(62 \mathrm{~J}, \ldots, 72 \mathrm{M})$ illustre la façon dont les éléments successifs d'une interlocution s'organisent hiérarchiquement. ben 3 bibliothèques on doit mettre termine le processus de résolution du problème mis en œuvre par les enfants, mais pas la tâche qui leur est demandée. En effet, il leur faut encore écrire la solution qu'ils viennent de découvrir. Cette activité va leur demander 9 tours de parole, sans compter les sourires, et plus d'actions élémentaires encore. Bien que ces différentes actions s'effectuent les unes derrière les autres, en concaténation, celleci représente en fait la projection linéaire de structures qui sont en réalité hiérarchisées (Clark, 1996). Par exemple, en 63M, Marc dit bi-bli-o-thèque, mais écrit $b i$-li-o-thèque. D'où la demande de correction formulée par Jean. Mais cette formulation de la requête de correction est elle-même erronée. Elle entraîne une auto-correction de la part de Jean, etc. Une représentation «correcte » du déroulement de l'activité devrait ainsi exprimer les différentes structures insérées ou hiérarchiques qui s'emboîtent successivement dans l'interlocution, comme le fait la formule suivante où « 3 » indice l'introduction de la requête d'autocorrection, « 5 » l'erreur de Marc selon Jean, « 7 » l'autocorrection de Jean, etc.

${ }_{1}\left(62 \mathrm{~J}_{2}\left(63 \mathrm{M}_{3}\left(64 \mathrm{~J}_{4}\left(65 \mathrm{M}_{5}\left(66 \mathrm{~J}_{6}\left(67 \mathrm{M}_{7}\left(69 \mathrm{~J}_{8}\left(70 \mathrm{M}_{9}(71 \mathrm{~J}, 72 \mathrm{M})_{9}\right)_{8}\right)_{7}\right)_{6}\right)_{5}\right)_{4}\right)_{3}\right)_{2}\right)_{1}$ 
Comme la concaténation est la structure liminaire de l'interlocution, d'un point de vue à la fois logique, génétique ( $C f$. Trognon \& Sorsana, 2005 ; dans l'introduction la référence aux proto-conversations) et fonctionnel (Schegloff, 1991 ; Trognon \& Batt, 2010 ; et dans la partie précédente, le deuxième paragraphe de 1)), il est nécessaire de lui associer une représentation (une forme) logique concordante. Cela explique que le raisonnement qui se déploie parallèlement au déroulement de l'interlocution soit représenté en logique interlocutoire grâce à la méthode de la déduction naturelle et plus précisément au calcul des séquents. En effet, cette méthode présente les connecteurs logiques comme des schémas d'inférence donc comme des processus. Cependant, dans un grand nombre d'interactions, les interlocuteurs conduisent des raisonnements non monotones. "Such reasoning is based on what may generally be expected to happen, subject to exceptions, in a familiar kind of situation. This whole area of study is associated with default inferences and abductive reasoning " (Walton, 2007 : 185). Comme Hintikka (2007) le souligne avec juste raison, les inférences non monotones ne sont pas moins logiques que les inférences monotones: ce sont simplement des inférences (logiques) reposant sur des prémisses provisoires qui sont supposées vraies jusqu'à plus ample information. Les déductions naturelles de la logique interlocutoire seront donc a priori non monotones, et seulement a posteriori monotones (Trognon \& Batt, 2010 ; Trognon \& Coulon, 2001). Ainsi adaptées, les déductions naturelles s'appliqueront en logique interlocutoire aux interprétations logiques des particules qui relient les énoncés d'une langue naturelle, ce qui augmente d'autant l'alphabet sur lequel travaille le langage de la logique interlocutoire.

\subsection{La production des événements interlocutoire est distribuée}

Enfin, la production de l'interlocution est fondamentalement distribuée, même à un niveau très élémentaire.

Voyez par exemple 29Md (ben faut faire) dans l'extrait. Avec cet énoncé, $\mathrm{M}$ conclut (c'est un assertif) sa réflexion. Mais l'acte de langage qu'il accomplit, une proposition, est incomplet. Bien que la force de l'acte, mi-directive, micommissive (Trognon, Sorsana \& Batt, 2008 ; Walton, 2007), cependant indirectement exprimée, soit accessible, son contenu propositionnel est vide. Il va être instancié d'abord par Jean en 30J, donc par l'auditeur de 29Md, mais sous la forme d'une question propositionnelle, puis par Marc répondant à la question et instanciant à son tour sous la forme d'une disjonction un contenu propositionnel différent.

Le contenu propositionnel de $29 \mathrm{Md}$ est ainsi le produit d'une activité conjointe des interlocuteurs. Leur collaboration dans l'instanciation d'un contenu propositionnel à $29 \mathrm{Md}$ les conduit à avancer deux alternatives, l'une portée par Jean, l'autre portée par Marc, ce qui du coup les entraîne dans un jeu de dialogue de délibération que nous qualifierions de délibération productive ${ }^{(2)}$. La qualité de la délibération s'exprime dans la formulation de Marc, qui expose «son » al-

(2) Nous verrons pourquoi bientôt quand nous rendrons compte de la validité logique du «tournant » donné par Jean à sa pensée. 
ternative d'une manière non conflictuelle (ou peut-être fois aussi), la disjonction (inclusive) ou associant les alternatives sans les opposer ${ }^{(3)}$, signal réitéré grâce, d'une part, à la modalité du possible assortie à l'option multiplicative et, d'autre part, à l'adverbe aussi qui, additionnant des deux procédés, ne barre pas l'option de Jean. Aussi l'acte de langage accompli par Marc s'apparente-t-il plutôt à une invite faite à Jean de réfléchir au procédé proposé par Marc, ce que Jean fera d'ailleurs dans les tours de parole suivants. Les processus illocutoires et propositionnels de la délibération qui s'instancient à ce moment-là convergeront ensuite en marquant la coopérativité de l'interaction.

Puisqu'à tous les niveaux de l'interlocution, les objets qui s'y découvrent sont produits conjointement par les interlocuteurs, certes à des degrés divers (Trognon, Batt, Sorsana et alii, 2010 ; Trognon \& Batt, 2010 ; Trognon \& Bromberg, 2007) ${ }^{(4)}$; nous préférerons la méthode dialogique à toute autre méthode d'exposition des processus intersubjectifs régissant l'interlocution.

D'un point de vue psycho-socio-discursif, les méthodes dialogiques sont nécessaires tant pour expliquer la production des forces illocutoires que pour expliquer la production des contenus propositionnels, pour expliquer la production de preuves logiques de même que l'établissement de vérités matérielles (Cf. Trognon \& Batt, 2010). Evidemment, nous ne prétendons pas que la méthode dialogique soit la seule valable. Après tout, le dialogue intérieur est un fait avéré, même s'il est dérivé (Wertsch, 1979 ; après Vigotsky, 1934). D'autres méthodes sont tout aussi pertinentes d'un point de vue logique. Mais si toutes ces méthodes sont d'égale pertinence d'un point de vue logique, il reste à démontrer qu'elles le sont aussi d'un point de vue psychologique. Vu les données dont on dispose aujourd'hui, cela est hautement improbable (Trognon \& Batt, 2010).

\subsection{La préparation de l'interlocution pour l'analyse : le tableau d'analyse interlocutoire}

Walton emploie une jolie expression pour décrire le rôle des actes de langage dans la constitution des mouvements d'un dialogue. Il écrit : « The speech act represents a kind of move that is made at a local level in a dialog » (138). Entre les uns et les autres, le lien n'est évidemment pas direct. Mais comme les premiers sont l'ossature de la construction, toute analyse doit débuter par l'identification des actes de langage littéralement représentés dans les énoncés de l'interlocution. La première étape de l'analyse consiste ainsi selon une problématique semblable à porter la transcription du discours de chacun des deux interlocuteurs sur deux colonnes séparées d'un même tableau d'analyse interlocutoire (tableau 1). En distribuant ainsi le discours, nous matérialisons la propriété de dialogicité. Un des multiples intérêts de cette représentation est de préparer les « données » pour une analyse approfondie du système dialectique, par l'étude

(3) $C f$. plus loin, la règle (D. or)

(4) Le degré de dialogisme d'une interlocution est d'autant plus élevé que l'intentionnalité mise en œuvre est plus collective. Mais l'intercompréhension d'un acte de langage élémentaire impose déjà une activité conjointe (Schegloff, 1991 ; Trognon, 2002 ; Trognon \& Batt, 2010). 
du passage de sa forme textuelle à son organisation psychosociodiscursive. Chaque colonne est subdivisée en sous-colonnes. Sur la première sous-colonne, sont inscrits les énoncés - composants élémentaires des contributions discursives - ordonnés en fonction de l'ordre de leur apparition. Nous tenons ainsi à respecter la propriété de séquentialité du dialogue car c'est elle qui forme l'assise empirique de tous les événements interlocutoires. Ces éléments alors ordonnés reçoivent ensuite leurs interprétations illocutoires. La force de l'acte de langage définie par son but apparaît dans la seconde sous-colonne, le contenu propositionnel dans la troisième. Sur la colonne du milieu, située entre les deux colonnes des interlocuteurs, est portée, pas à pas, l'analyse des relations inter-énoncés au fur et à mesure de leur apparition. Voici ce que donne une telle représentation pour la séquence cognitivement féconde dans la découverte par les enfants de la solution au problème que comporte notre extrait.

\begin{tabular}{|c|c|c|c|c|c|c|}
\hline & & $(\exists n) K$ & $\begin{array}{r}\text { Consigne } \\
C / R=672 \\
C / E=32 \\
E / B=7 \\
\text { perimentateurKjean\&m } \\
\text { sideratum }=(B)\end{array}$ & $\begin{array}{l}\operatorname{larc}(B / R=n) \\
/ R=n)\end{array}$ & & \\
\hline & & & $\begin{array}{l}\text { Demande de } \\
\text { confirmation de } \\
\text { diviser par } 32\end{array}$ & $\begin{array}{l}30 \mathrm{~J} \\
\text { faut diviser } \\
\text { par } 32 \text { ? (il le } \\
\text { regarde) }\end{array}$ & Directif & $\begin{array}{l}\text { Faut diviser? } \\
\text { /32? }\end{array}$ \\
\hline $\begin{array}{l}31 \mathrm{M} 1 \\
\text { ou peut-être fois } \\
\text { aussi }\end{array}$ & Assertif & $\begin{array}{l}\text { Peut-être: } \\
\text { modalité } \\
\text { ontique } \\
\text { fois }\end{array}$ & $\begin{array}{l}\text { Proposition } \\
\text { alternative } \\
\text { (ou peut-être) } \\
\text { non exclusive } \\
\text { (aussi) }\end{array}$ & & & \\
\hline $\begin{array}{l}31 \mathrm{M} 2 \\
\text { parce que } 32 \text { fois } \\
\text { combien }\end{array}$ & & 32 fois $n$ & $\begin{array}{l}\text { Justification } \\
\text { (parce que) }\end{array}$ & & & \\
\hline & & & $\begin{array}{l}\text { Proposition } \\
\text { alternative (à } \\
\text { moins que) }\end{array}$ & $\begin{array}{l}32 \mathrm{~J} \\
\text { A moins qu'on } \\
\text { arrive à chaque } \\
\text { fois à } 32\end{array}$ & Assertif & $n=32$ \\
\hline $\begin{array}{l}33 \mathrm{M} \\
\text { Attends }\end{array}$ & Directif & & Ordre & & & \\
\hline & & & & $\begin{array}{l}\text { 34J1 } \\
32 \text { "fois" } 7\end{array}$ & Assertif & $32 \times 7$ \\
\hline & & & & $\begin{array}{l}34 \mathrm{~J} 2 \\
\text { (Calcule)... } \\
\text { Mmh... possible }\end{array}$ & Assertif & \\
\hline & & & & $\begin{array}{l}34 \mathrm{~J} 3 \\
\text { par étagères tu } \\
\text { peux en mettre } \\
200 \ldots 224\end{array}$ & Assertif & $\begin{array}{l}\mathrm{C} / \mathrm{E}(\mathrm{B})=224 \\
\text { (ambigüité entre } \\
\text { étagère et } \\
\text { bibliothèque) }\end{array}$ \\
\hline
\end{tabular}




\begin{tabular}{|c|c|c|c|c|c|c|}
\hline & & & Déduction & $\begin{array}{l}34 \mathrm{~J} 4 \\
\text { alors il y a qu'a } \\
\text { enlever à } \\
\text { chaque fois } 224\end{array}$ & Assertif & $\begin{array}{l}672-n \text { fois } \\
224=0\end{array}$ \\
\hline $\begin{array}{l}35 \mathrm{M} 1 \\
\text { Par étagère... } 32\end{array}$ & Assertif & $C / E=32$ & & & & \\
\hline $\begin{array}{l}\text { 35M2 } \\
\text { Ah ouais }\end{array}$ & Expressif & & Accord de M & & & \\
\hline \multirow[t]{2}{*}{$\begin{array}{l}35 \mathrm{M} 3 \\
\ldots \text { Ben } 600\end{array}$} & Assertif & $\begin{array}{l}\text { Ben: scande } \\
\text { une } \\
\text { déduction }\end{array}$ & & & & \\
\hline & & & $\begin{array}{l}\text { Accord de J } \\
\text { Correction et } \\
\text { levée de } \\
\text { l'ambigüité }\end{array}$ & $\begin{array}{l}36 \mathrm{~J} \\
224 \text { ouais ! par } \\
\text { BIBLIOTHEQUE } \\
\text { (accentué) }\end{array}$ & Assertif & $C / B=224$ \\
\hline $\begin{array}{l}\text { 37M1 } \\
\text { Ah oui }\end{array}$ & $\begin{array}{l}\text { Expres- } \\
\text { sif }\end{array}$ & & Accord de M & & & \\
\hline $\begin{array}{l}37 \mathrm{M} 2 \\
\text { Ben fais sur le } \\
\text { brouillon }\end{array}$ & Directif & $\begin{array}{l}\text { Ben : scande } \\
\text { la poursuite } \\
\text { du travail } \\
\text { Faire sur le } \\
\text { brouillon } \\
\text { (Marc, y) }\end{array}$ & $\begin{array}{l}\text { Requête de M } \\
\text { à J de } \\
\text { poursuivre leur } \\
\text { tâche }\end{array}$ & & & \\
\hline \multirow[t]{2}{*}{$(\ldots)$} & & & & $(\ldots)$ & & \\
\hline & & & & $\begin{array}{l}58 \mathrm{~J} \\
\text { ben ... on met... } \\
\text { il faut } 3 \\
\text { étagères! }\end{array}$ & Assertif & $\begin{array}{l}\text { Modalité } \\
\text { déontique } \\
\text { B/R = } 3\end{array}$ \\
\hline \multirow[t]{2}{*}{$\begin{array}{l}59 \mathrm{M} \\
3 ! ?\end{array}$} & Assertif & & & & & \\
\hline & & & & $\begin{array}{l}60 \mathrm{~J} \\
3 !\end{array}$ & Assertif & \\
\hline \multirow[t]{2}{*}{$\begin{array}{l}61 \mathrm{M} \\
3 \text { bibliothèques }\end{array}$} & Assertif & & & & & \\
\hline & & & $\begin{array}{l}\text { Desideratum } \\
=3\end{array}$ & $\begin{array}{l}62 \mathrm{~J} \\
3 \text { bibliothèques! }\end{array}$ & Assertif & $B / R=3$ \\
\hline
\end{tabular}

\section{Tableau 1. Tableau analyse interlocutoire}

La représentation précédente fournit le «contenu » pragmatico-sémantique quasi-explicitement exposé au fur et à mesure des contributions successives des interlocuteurs. Les trois premières colonnes correspondent aux productions de $\mathrm{M}$; les trois dernières aux productions de J. Chaque groupe présente successivement le discours matériellement énoncé, l'acte illocutoire qu'il accomplit, puis le contenu propositionnel de cet acte, La colonne du milieu présente la fonction conversationnelle de l'acte. Les contenus propositionnels sont exprimés en langage arithmétique. Les formules correspondantes explicitent les prémisses et les conclusions des raisonnemnets que les enfants conduisent tandis 
qu'ils conversent. Cela est fort bien illustré par la séquence 34J3-34J4-36J. $34 \mathrm{~J} 3$ énonce le résultat du calcul dont $34 \mathrm{~J} 2$ accompagne l'accomplissement. Le contenu propositionnel de cette assertion est $\mathrm{C} / \mathrm{E}[\mathrm{B}]$ : littéralement $\mathrm{J}$, une nouvelle fois victime de l'ambiguité étagère/bibliothèque, dit « étagère » en pensant (si l'on peut dire) «bibliothèque ». Des sous-tableaux enrichiront ensuite ses lignes afin de déployer leur structures dialectiques en termes de jeux sémantiques (Hintikka, 1994) et de jeux de dialogues (Carlson, 1983 ; Hintikka, 1983, 1984 ; Walton, 2007). Grâce à cet enrichissement, nous pourrons rendre compte de la production logique d'une interlocution. Nous aborderons cet aspect plus loin quand nous étudierons la découverte de la solution du problème par les enfants.

\subsection{Le séquent de dialogue comme composant élémentaire de l'analyse de l'interlocution}

Le concept de séquent de dialogue résume la problématique que nous venons d'exposer intuitivement. Ce terme est emprunté à la logique, où il désigne un couple noté $\Gamma \vdash F$. « $\Gamma$ est un ensemble fini de formules. [il] représente les hypothèses que l'on peut utiliser. Cet ensemble s'appelle aussi le contexte du séquent. $F$ est une formule. C'est la formule que l'on veut montrer. On dira que cette formule est la conclusion du séquent ». (David, Nour, \& Raffali, 2003, p. 24). En élargissant la notion de séquent aux illocutions et en s'inpirant d' une perspective ouverte par Carlson (1983), chaque énoncé est représenté en logique interlocutoire par une expression de la forme qui suit: $<\mathrm{Mi},\{\mathrm{Mi}-\mathrm{k}\},\{\mathrm{Mi}-$ $\mathrm{k}\} \vdash \mathrm{Mi}, \mathrm{RD}, \mathrm{DG}>$. Mi est le mouvement conversationnel accompli par l'énoncé examiné. \{Mi-k\} est l'ensemble de tous les mouvements conversationnels qui précèdent le mouvement Mi et auxquels celui-ci enchaîne. Mi peut alors être conçu comme une conclusion qui découle des prémisses $\{\mathrm{Mi}-\mathrm{k}\}$. L'enchaînement qui conduit de $\{\mathrm{Mi}-\mathrm{k}\}$ à Mi et que l'on représente par le schéma $\{\mathrm{Mi}-\mathrm{k}\} \vdash$ Mi est un séquent de dialogue. RD est l'ensemble des règles de dialogue utilisées par les interlocuteurs pour accomplir leurs mouvements dans le dialogue. Enfin DG est le(s) jeu(x) de dialogue joué(s) par les interlocuteurs dans le fragment étudié. Carlson (1983) illustre la notion de séquent de dialogue à partir du dialogue suivant :

Contexte : A se rend au stade pour assister à une finale de saut à la perche entre Jack et Bob. Mais A arrive trop tard : le match est terminé. Apercevant son ami Bob, Jack lui demande :

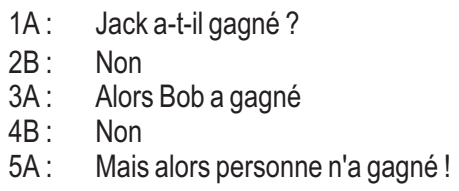

$2 \mathrm{~B}$ est une réponse à $1 \mathrm{~A}$. Le séquent de dialogue qui « engendre » cette réponse est $\{1 \mathrm{~A}\} \vdash 2 \mathrm{~B}$. La règle de dialogue qui permet de dériver $2 \mathrm{~B}$ de $1 \mathrm{~A}$ est une règle de disjonction typique des jeux de dialogue question-réponse. $3 \mathrm{~A}$ est issue des «pensées » entretenues par A (si quelqu'un a gagné c'est Jack ou Bob) et de l'information transmise par 2B (Jackn'a pas gagné) travaillées ensemble 
grâce à la règle logique d'élimination de la disjonction : $\{1 \mathrm{~A}, 2 \mathrm{~B}\} \vdash 3 \mathrm{~A}$. 5A se déduit grâce à la règle d'élimination de la disjonction ou de la règle classique de l'absurde qui s'applique pour relier les pensées entretenues par A (si quelqu'un a gagné c'est Jack ou Bob, et Jackn'a pas gagné) à l'information transmise par 4B (Bob n'a pas gagné). Le séquent de dialogue représentant la " forme logique » de l'interlocution précédente est donc $\{1 \mathrm{~A}, 2 \mathrm{~B}, 3 \mathrm{~A}, 4 \mathrm{~B}\} \vdash 5 \mathrm{~A}$.

\section{Les processus psycho-socio-discursifs impliqués dans la découverte de la solution du problème par les enfants}

\subsection{Une interlocution coopérative centrée sur le problème}

A observer les colonnes du tableau 1, il apparaît qu'il faut une trentaine de tours de parole à chacun des deux interlocuteurs pour parvenir à une conclusion commune. On peut donc affirmer qu'au cours de cet échange la distribution de la parole entre les enfants est homogène.

De $25 \mathrm{M}$ à $29 \mathrm{M}$, c'est ensemble que les enfants reformulent les éléments de la consigne pertinents pour la résolution du problème. En 29M, après avoir réaffirmé un mouvement de dialogue intérieur (ouais) où s'enchaîne un raisonnement global (pis), Marc introduit une déduction sur le mode déontique ( $\mathrm{il}$ faut), sous-entendant ainsi que seule la solution qu'il s'apprête à énoncer est envisageable. Il ne dévoile pas son idée, c'est Jean qui en 30J, complète sa proposition, sans toutefois l'imposer mais en demandant une confirmation sur le mode verbal (formulation interrogative) et non verbal (regards) à son partenaire. Jean et Marc instaurent une relation symétrique : à la tempérance de Jean répond celle de Marc qui, à son tour en $31 \mathrm{M}$, à l'aide d'une modalité ontique (peut-être) propose une alternative (ou) tout en validant partiellement l'idée de Jean (aussi).

En 32J, Jean émet une restriction (à moins que). En 33M, Marc l'interrompt, lui ordonne de suspendre son raisonnement (attends). Jean n'obtempère pas. La relation des deux enfants se dessine plus précisément en 37M2, 38J, 39M, 40J. Cette courte séquence comporte une requête $(37 \mathrm{M} 2,(\ldots), 39 \mathrm{M})$ exprimée par un énoncé impératif (fais sur le brouillon), suivie de sa satisfaction (40J). Tout se passe comme si Marc avait pris un ascendant (au moins technique : c'est lui qui écrit sur la feuille) sur Jean puisque les deux actes directifs de la séquence sont énoncés par Marc à l'attention de Jean. Mais l'analyse des modalités semble démontrer que le comportement discursif modéré de Jean contribue à la collaboration des deux enfants. Nouveau signe que les enfants ne sont pas en compétition dans cette interaction.

Ainsi, on ne relève pas de trace de conflit entre les enfants qui adoptent une attitude collaborative en s'auto-corrigeant mutuellement lorsque des erreurs apparaissent, comme en $36 \mathrm{~J}$, ou encore en utilisant parfois des formules visant à protéger la face de l'autre comme en 30J, 31 M, 32J. Dans cette séquence, c'est bien à la résolution conjointe d'un problème dans une situation d'interaction collaborative directe que nous assistons. 


\subsection{Le processus cognitif de résolution du problème et sa distribution}

Le climat de l'interaction nous autorise à concentrer notre analyse sur les contenus propositionnels de l'interlocution afin de faire ressortir les raisonnements conduits par les enfants pour résoudre le problème.

\subsubsection{L'énoncé du problème}

La consigne comporte un ensemble d'informations et une question. Elle décrit un monde dans lequel des objets appartiennent à des sous-ensembles de ce monde. Ainsi : C est l'ensemble «cassettes »; E est l'ensemble « étagères »; B est l'ensemble « bibliothèques »; R est l'ensemble « tout le rangement des cassettes ». On observe que chaque élément de $\mathrm{C}$ (les cassettes) appartient à un élément de $\mathrm{E}$ (une étagère), autrement dit que les éléments de E sont des sous-ensembles de C. De même : les éléments de B sont des sous-ensembles de E, et les éléments de R sont des sous-ensembles de B. Cela nous donne les trois prémisses avancées dans l'énoncé du problème. Elles sont écrites en langage du calcul des prédicats, comme des propositions d'identité. Cela nous donne les trois prémisses avancées dans l'énoncé du problème.

$\mathrm{C} / \mathrm{E}=32$ : le nombre de cassettes contenues dans $\mathrm{E}$ est égal à 32 ;

$\mathrm{E} / \mathrm{B}=7$ : le nombre d'étagères contenues dans $\mathrm{B}$ est égal à 7 ;

$C / R=672$ : le nombre de cassettes que le rangement total comporte est égal à 672 .

La question de la consigne est une question catégorielle «(...) de combien de bibliothèques a-t-il besoin pour ranger ces cassettes? » qui appelle le desidera$\operatorname{tum}^{(5)}$ : l'expérimentateur sait que Marc et Jean savent « combien de bibliothèques » le commerçant a besoin pour ranger ses 672 cassettes. Par conséquent l'information visée est « le commerçant a besoin de $\mathrm{n}$ bibliothèque(s) pour ranger ces 672 cassettes », enchâssée dans « Marc et Jean savent combien (...) », enchâssée dans « l'expérimentateur sait que (... (...)) ». Cela donne :

$$
(\exists \mathrm{n}) \mathrm{K}_{\text {expérimentateur }} \mathrm{K}_{\text {Marc et Jean }}(\mathrm{B} / \mathrm{R}=\mathrm{n})^{(6)} \text {. }
$$

(5) Le desideratum d'une question est la demande de la personne qui pose la question. Hintikka (1976, $1981 ; c f$. également Carlson, 1983), à qui nous empruntons notre analyse des questions, définit une question comme l'accomplissement d'un acte de langage optatif dont le contenu propositionnel est l'état mental satisfait par l'information demandée. Une question se glose donc : Fais en sorte (optatif) que je sache (attitude propositionnelle (épistémique) du demandeur) que 3 bibliothèques sont nécessaires pour ranger toutes les cassettes (information demandée). La question posée aux enfants par l'expérimentateur est une question catégorielle, c'est à dire une question pour laquelle «le choix porte sur les valeurs d'une variable quantifiée » (Hintikka, 1976, p. 60). Les enfants se posent quant-à-eux des questions propositionnelles, c'est-à-dire des «questions où le choix a trait à des propositions » ( $i b i$ dem,p. 60). Exemple : $30 \mathrm{~J}$ et $31 \mathrm{Ma}$ sont des questions propositionnelles. Notez que de $30 \mathrm{~J}$ à $31 \mathrm{Ma}$ la réponse attendue passe d'une alternative absolue (oui/non) à une alternative relative (« fois » et-ou « diviser », ce qui nous renvoit au climat de l'interlocution, donc à la remarque que le contenu propositionnel contribue pour une part au climat de l'interlocution.

(6) La question posée est une question d'examen : le questionneur veut savoir si le questionné sait (ce que le questionneur sait). Cf. Carlson, 1983, Walton, 2007. 


\subsubsection{Le raisonnement mis en œuvre par les enfants}

On observe que chacun des deux enfants se pose une question catégorielle identique en surface : combien y a-t-il d'étagères ? Mais leur question ne porte pas sur le même référent ${ }^{(7)}$. Le desideratum de la question de Jean correspond au nombre total d'étagères nécessaires pour ranger 672 cassettes $(E / R)$ «combien y a-t-il besoin d'étagères pour ranger ces cassettes?". Le desideratum de la question de Marc se déduit, quant à lui, par la réponse concluante qu'il apporte lui-même : "-combien y a-t-il d'étagères? - il y a 7 étages ». Marc s'intéresse donc au nombre d'étagères par bibliothèque $(\mathrm{E} / \mathrm{B})$. On note leur convergence pour poser comme prémisse dans leur raisonnement respectif le fait qu'il y a 7 étagères par bibliothèque. On observe aussi leur divergence (relative, car comme nous l'avons remarqué dans l'analyse précédente, Marc ne réfute pas le choix de Jean) sur le choix de l'opération : la multiplication pour Marc, la division pour Jean. On accède ainsi à la distribution des processus cognitifs qui sont en cours avant $34 \mathrm{~J}$. Le raisonnement que Jean semble mener plus ou moins explicitement est : combien a-t-il besoin d'étagères pour ranger ses cassettes? Sachant qu'il a 672 cassettes à ranger et sachant qu'il peut en ranger 32 par étagère, il lui faut 672/32 = 21 étagères pour s'acquitter de sa tâche. Le raisonnement de Marc semble être : Sachant qu'il y a 7 étagères par bibliothèque et que l'on peut mettre 32 cassettes dans chaque étagère, alors il y a 7 fois 32 cassettes par bibliothèque. Si l'on poursuit le raisonnement de chacun jusqu'à son aboutissement, on obtient par deux voies différentes, la réponse concluante à la question catégorielle de l'expérimentateur. C'est ce qui est porté sur le tableau 2 (page suivante).

Au vrai, la divergence entre Jean et Marc quant au premier pas à faire pour résoudre le problème est antérieure à la séquence que nous examinons présentement (Trognon, Saint-Dizier \& Grossen, 1999).

\section{[Marc lit l'énoncé à haute voix] \\ 1J: $\quad$ Ahil faut diviser \\ 2M: peut-être pas \\ 3J: $\quad$ presque \\ (...)}

16J: $\quad$ attends...on divise par 32

[Silence, ils regardent tous les deux la feuille de l'énoncé]

Comme on s'en aperçoit immédiatement à la lecture de ce court extrait, Jean projette de faire une division, à peine a-t-il lu l'énoncé du problème. Marc hésite, cependant sans être capable de formuler une autre proposition ou d'objecter. Ces alternatives stratégiques encore balbutiantes ne renvoient pas seulement aux processus mentaux des enfants. Elles réfléchissent aussi le contexte cognitif dans lequel ces processus opèrent, lequel est créé par une énonciation

(7) L'ambiguité concerne ici l'ensemble contenant les étagères : le « tas » de cassettes pour Jean, une bibliothèque pour Marc. Mais le corpus comporte une seconde ambiguité : «étagères » signifiant tantôt «étages » (planches) tantôt «bibliothèques ». Comme on le constate en retournant au corpus, cette seconde ambiguité gêne les enfants tout au long de leur travail et les oblige à contrôler constamment leurs énoncés ( $C f$. Trognon, Saint-Dizier \& Grossen, 1999, où cette ambiguité est analysée plus en détail). 


\begin{tabular}{|c|c|c|c|c|c|}
\hline \multicolumn{3}{|r|}{ MARC } & \multicolumn{3}{|c|}{ JEAN } \\
\hline \multicolumn{3}{|c|}{$\begin{array}{l}\text { 25M-27M1 : combien y a-t-il d'étagères? } \\
\mathrm{E} / \mathrm{B}=?\end{array}$} & \multicolumn{3}{|c|}{$\begin{array}{l}26 \mathrm{~J} \text { : combien a-t-il besoin d'étagères pour ranger } \\
\text { ses cassettes } \\
\mathrm{E} / \mathrm{R}=\text { ? }\end{array}$} \\
\hline \multicolumn{3}{|c|}{$\begin{array}{l}27 \mathrm{M} 2 \text { : il y a } 7 \text { étages (sous entendu par } \\
\text { bibliothèque) } \\
\mathrm{E} / B=7\end{array}$} & \multicolumn{3}{|c|}{$\begin{array}{l}28 \mathrm{~J} \text { : il y a } 7 \text { étages (sous entendu par } \\
\text { bibliothèque) } \\
\mathrm{E} / \mathrm{B}=7\end{array}$} \\
\hline \multicolumn{3}{|c|}{$\begin{array}{l}27 \mathrm{M} 3 \text { : on peut mettre } 32 \text { dans une (sous entendu } \\
\text { une étagère) } \\
\mathrm{C} / \mathrm{E}=32\end{array}$} & \multicolumn{3}{|c|}{ 30J : faut diviser par $32 ?$} \\
\hline \multicolumn{6}{|c|}{$31 \mathrm{M}$ : ou peut-être fois aussi } \\
\hline \multicolumn{6}{|c|}{ Raisonnements complets possibles } \\
\hline \multicolumn{3}{|c|}{$\begin{array}{l}\text { Raisonnement possible en débutant par une } \\
\text { opération de démarrage = multiplication }\end{array}$} & \multicolumn{3}{|c|}{$\begin{array}{l}\text { Raisonnement possible en débutant par une } \\
\text { opération de démarrage = division }\end{array}$} \\
\hline \multirow{2}{*}{$\begin{array}{c}32 \times 7=22 \\
672\end{array}$} & & démarrage en multipliant & \multirow[t]{2}{*}{672} & 32 & démarrage en divisant \\
\hline & 224 & poursuite en divisant & & 21 & poursuite en divisant \\
\hline \multicolumn{3}{|c|}{$\begin{array}{l}\text { Les enfants opteront finalement pour } \\
\text { ce raisonnement }\end{array}$} & \multicolumn{3}{|c|}{$\begin{array}{l}\text { Raisonnement commencé par Jean puis } \\
\text { abandonné par lui }\end{array}$} \\
\hline
\end{tabular}

Tableau 2. Processus de raisonnement de chaque enfant avant $34 J$.

qui qualifie incomplètement le problème (défini comme un problème de multiplication) posé aux enfants au regard des moyens que sa solution requiert : deux divisions ou une multiplication et une division ${ }^{(8)}$. Si les enfants balancent entre deux stratégies c'est donc aussi parce que la consigne (l'expérimentateur) leur indique un procédé alors que le problème leur en impose un autre. Le contexte expérimental instaure ainsi un conflit entre la demande de l'expérimentateur (faire des multiplications) et la demande « objective » du problème. Ce conflit devrait être confronté au modèle du conflit socio-cognitif(Sorsana, 1999) puisque les enfants doivent satisfaire une demande sociale (la demande de l'expérimentateur) et une demande cognitive (la demande du problème) qui sont discordantes. En tout cas, ce conflit appartenant au contexte se traduit aussi dans la relation instaurée entre les enfants. Le proposition de Marc est plutôt orientée vers la satisfaction de la demande sociale tandis que celle de Jean est plutôt orientée vers la satisfaction de la demande « cognitive». Les enfants, qui gèrent très prudemment leur relation, se trouvent dès lors engagés dans un jeu de dialogue délibératif (Walton, 2007).

(8) Les enfants obéiront littéralement à la consigne en trouvant une solution qui accomplit une division en n'effectuant que des multiplications. Ce qui est tout de même assez extraordinaire! Cf. supra en conclusion. 


\subsubsection{Logique de la solution trouvée par les enfants (le moment logiquement fécond de l'interlocution)}

Pour ne pas imposer au lecteur un effort disproportionné, nous exposerons la logique de la solution trouvée par les enfants en commençant par sa présentation axiomatique, à la Hilbert. Cette méthode d'exposition ne restitue pas la processualité du raisonnement. Mais, équivalente aux autres méthodes d'un point de vue logique, elle a l'avantage de simplifier l'exposition des objets auxquels on se réfèrera de toute façon. $C^{\prime}$ est donc seulement dans un second temps que nous proposerons une présentation fondée sur une méthode plus respectueuse du processus de production du raisonnement mené par les enfants, présentation que nous compléterons encore par des remarques plus informelles.

\section{a) Reconstruction axiomatique de la logique du raisonnement conduit par les enfants}

$\begin{array}{llll}\text { 1. } & C / E=32 & \text { Prémisse } & \\ \text { 2. } & E / B=7 & \text { Prémisse } & \\ \text { 3. } & C / R=672 & \text { Prémisse } & \\ \text { 4. } & C / B=C / E \times E / B & \text { Vérité algébrique } & \\ \text { 5. } & C / B=32 \times E / B & \text { =Elim-1,4 } & \text { Plan de Marc. Desideratum de sa question } \\ \text { 6. } & C / B=32 \times 7 & =E l i m-2,5 & \text { Réponse implicite de Jean à Marc } \\ \text { 7. } & 32 \times 7=224 & \text { Arithmétique } & \text { Multiplication effectuée par Jean } \\ \text { 8. } & C / B=224 & =E-6,7 & \\ \text { 9. } & C / R=C / B \times B / R & \text { Vérité algébrique } & \\ \text { 10. } \quad C / R=224 \times B / R & =E-8,9 & \\ \text { 11. } \quad 672=224 \times B / R & =E-3,10 & \\ \text { 12. } & 672=224 \times 3 & \text { Arithmétique } & \text { Division effectuée par jean } \\ \text { 13. } & B / R=3 & =E-11,12 & \text { Solution du problème }\end{array}$

Les nombres portés dans la première colonne désignent les différentes formules utilisées par les enfants. Ils désignent aussi les différentes étapes du raisonnement. Les formules sont des énoncés d'identité, donc des propositions. Leur contenu est donné dans la seconde colonne. La troisième colonne définit le statut conceptuel des formules. Certaines sont des prémisses données dans l'énoncé du problème. D'autres sont des déductions, par exemple les formules produites grâce aux règles d'élimination $(5,6,8,10,11,13)$. D'autres encore sont des connaissances mathématiques. La quatrième colonne rapporte les différentes formules à leur rôle dans l'interlocution. 5 est le desideratum de la question posée par Marc, 6 est la réponse implicite de Jean à cette question, 7 représente le calcul que Jean effectue plus ou moins en pensée, plus exactement dans une pensée plus ou moins affleurante dans l'énonciation : « 7 fois 32 » (Jean satisfait la requête de Marc, il fait combien fois), ... «14» (Jean pose le résultat de la multiplication de 2 par 7 ), «mmh ! (il considère le résultat de son calcul)... «possible» (exprime son atttitude propositionnelle vis-à-vis du résultat), «par étagères tu peux en mettre (= des cassettes) » (il énonce $C / B=224$, en confondant une fois de plus «étagère » et «blibliothèque »), « alors y a qu'à enlever à chaque fois 224 »(Jean propose une procédure pour trouver B/R). 


\section{b) Première tentative de reconstruction formelle du raisonnement conduit par les enfants en calcul des séquents}

Nous pouvons maintenant réexposer la démonstration précédente en calcul des séquents, c'est-à-dire avec une méthode plus respectueuse de la séquentialité de l'interlocution.

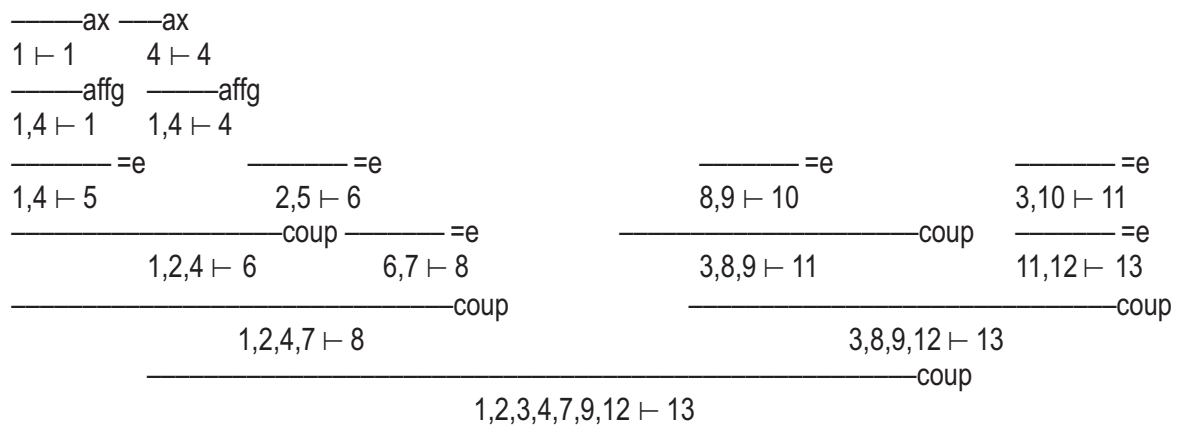

Première représentation en calcul des séquents du raisonnement conduit par les enfants

Pour suivre le déroulement du raisonnement des enfants, il faut lire l'inférence précédente de haut en bas ${ }^{(9)}$. La première ligne, dont les composants sont surlignés d'un trait qui se termine par une notation «ax» (= axiome) présente les formules de départ du raisonnement. Toutes n'ont pas été portées dans le schéma. Ces formules de départ représentent des prémisses du problème, par exemple $\mathrm{C} / \mathrm{E}=32$, est représentée par $(\mathrm{C} / \mathrm{E}=32) \vdash(\mathrm{C} / \mathrm{E}=32)$, ce qui est écrit ici 1 $\vdash 1$; $\mathrm{E} / \mathrm{B}=7$, est représentée par $2 \vdash 2$, et $\mathrm{C} / \mathrm{R}=672$, par $3 \vdash 3$; ou encore elles représentent des connaissances que les enfants importent dans leur raisonnement, par exemple la connaissance que le nombre de cassettes d'une bibliothèque est égal au nombre de cassettes contenues dans une étagère multiplié par le nombre d'étagères d'une bibliothèque, écrite ici $4 \vdash 4$ ou enfin elles représentent des calculs intermédiaires effectués par les enfants, par exemple $8 \vdash 8$, qui donne le nombre de cassettes contenues dans une bibliothèque $\mathrm{C} / \mathrm{B}=224$. Il suffit de se reporter à la présentation axiomatique de la logique du raisonnement pour retrouver le lexique des formules. Chaque fois que l'on descend d'un niveau dans le schéma d'inférence, on effectue une étape du raisonnement. Ainsi, par exemple, de $1,4 \vdash 5$ et de $2,5 \vdash 6$, les enfants déduisent le séquent $1,2,4 \vdash 6$ qui, conjoint au séquent $6,7 \vdash 8$ conduit à la conclusion provisoire $1,2,4,7 \vdash 8$. Les formules écrites au dessus d'un trait constituent les prémisses de l'infé-

(9) La représentation serait encore plus fidèle en décalant les lignes du calcul de façon à reproduire l'introduction progressive des propositions (en particulier des propositions arithmétiques). Pour ne pas dépasser la largeur de la page, on a omis de porter les origines de $8,9 \vdash 10$ et de $3,10 \vdash 11$. Elles se calculent de la même façon que sur le côté gauche. Notez que 9 est la clé de la solution, tout comme 4 de l'autre côté. C'est en intégrant ces deux «inventions » aux prémisses, ou en important ces connaissances arithmétiques dans les connaissances fournies par l'énoncé du problème, que les enfants résolvent le problème. 
rence. La formule écrite au dessous d'un trait est la conclusion qui se déduit de ces prémisses. L'expression à droite du trait représente la règle d'inférence qui a été utilisée pour passer des prémisses (ou des hypothèses) à la conclusion. Par exemple, de $\mathrm{C} / \mathrm{E}=32$ et de $\mathrm{C} / \mathrm{B}=\mathrm{C} / \mathrm{E} \times \mathrm{E} / \mathrm{B}$, les enfants déduisent $\mathrm{C} / \mathrm{B}=32 \times \mathrm{E} / \mathrm{B}$, qui conjoint avec $\mathrm{E} / \mathrm{B}=7$, donne aux enfants $\mathrm{C} / \mathrm{B}=32 \times 7$. En calcul des séquents : de $1,4 \vdash 5$ et de $2,5 \vdash 6$ les enfants déduisent $1,2,4 \vdash 6$ au moyen d'une règle dite de coupure signifiant intuitivement que si on déduit 5 de 1 et de 4 et 6 de 2 et de 5 alors de 1,2 et 4 on déduit $6: 5$ qui apparaît comme conclusion du premier séquent et comme prémisse du second séquent ayant servi d'intermédiaire à la déduction de 6 . Avec cette façon de faire, on tente d'accompagner de proche en proche, station après station pourrait-on dire, la réflexion des enfants. Le séquent terminal (13) est la réponse à la question de l'expérimentateur. Elle est déduite des données du problèmes (1, 2 et 3 ), des connaissances algébriques des enfants (4 et 9) et des deux opérations arithmétiques effectuées par Jean (7 et 12). Trois règles auront été utilisées plusieurs fois par les enfants. La règle de coupure aura été appliquée 5 fois. Les enfants auront également utilisé 6 fois la règle dite d'élimination de l'identité. Elle autorise à déduire (y)) de $(\mathrm{x})$ et de $\mathrm{x}=\mathrm{y}$. Enfin ils auront utilisé plusieurs fois la règle dite d'affaiblissement gauche, qui permet de réitérer des formules. Insistons encore une fois sur le fait que l'arbre précédent n'est qu'une approximation dont le seul mérite est de soutenir une comparaison avec ce qui s'est effectivement accompli dans l'interaction. A ce propos, il saute immédiatement aux yeux que le processus représenté par l'arbre n'est pas celui que les enfants ont suivi. En effet dans le dialogue des enfants 8 , i.e. $\mathrm{C} / \mathrm{E}(\mathrm{B})=224$, ne résulte pas de 1-2-4-7. Au contraire, on a l'impression que c'est une fois qu'il a effectué $32 \times 7$ que Jean découvre que ce produit est égal à $\mathrm{C} / \mathrm{E}(\mathrm{B})$. Du coup, les enfants n'utilisent pas à proprement parler la vérité algébrique 4, plutôt Jean puis Marc la découvrent en faisant le calcul $32 \times 7$. D'ailleurs, le nombre de cassettes par bibliothèque (indépendamment de l'ambiguité bibliothèque vs étagère) n'apparait pas dans le dialogue des enfants Dans le même ordre d'idées, le passage de 8 à 9 semble s'effectuer via l'introduction d'une implication (la vérité algébrique de la reconstruction axiomatique ci-dessus) implicite du genre : si 224 cassettes se rangent dans une bibliothèque $(224=\mathrm{C} / \mathrm{B})$ alors 672 cassettes se rangent en $x$ fois 224 cassettes donc dans $x$ bibliothèques, raisonnement qui est très imparfaitement rendu par la côté droit du séquent.

Il y a deux moments féconds dans le processus de résolution du problème. Chacun d'eux produit une donnée intermédiaire dont la combinaison avec l'autre donnée fera la solution : le nombre de cassettes contenues dans une bibliothèque (avant dernière ligne du séquent, côté gauche) et le nombre de bibliothèques nécessaires au rangement des cassettes ensuite (avant dernière ligne du séquent, côté droit).

Voyons ces deux séquences correspondantes en commençant par la première. Plusieurs événements s'y superposent. 4 (fois) représente le plan de Marc dans l'abstrait, un plan qui ne se constituera que rétrospectivement au travers de ses instanciations par Jean. La séquence exprimant ce plan n'expose pas complètement ses éléments, son dernier terme demandant l'information qui lui manque encore pour qu'il soit pleinement finalisé. 5 est la formule du desideratum de la 
question posée par Marc. 6 est l'instanciation en actes du desideratum. C'est la satisfaction de la question, la réponse implicite que Jean fait à Marc quand il essaie hors interaction, « en pensée » le plan de Marc et dont le résultat est 7. 8 identifie le calcul qui vient d'être effectué. Cette formule clôt la réalisation du projet de Marc, donc la première séquence et elle ouvre la seconde.

En effet, 7 fois $32 \ldots 14$ de $34 \mathrm{Ja}$ est un énoncé ambigu condensant un sens rétrospectif et un sens prospectif, un peu comme un mot-valise. On le formalise : «(...) [7) fois 32.. 14]». Selon une première lecture « 7 » appartient à la séquence qui précède 7 fois 32, c'est-à-dire à l'échange : Question indirecte (32 fois «combien »?) / Réponse (7). Selon une seconde lecture « 7 » appartient à l'opération « 7 fois $32 \ldots 14$ », où 14 est le résultat de la multiplication de 7 par 2 , la suite consistant à poser « 4 » et à retenir 1 , la dizaine. Dans les deux interprétations, 34Ja satisfait et donc adopte (même si c'est provisoirement, en acceptant de l'essayer) la proposition de Marc qu'il réalise en actes. Ce faisant, Jean abandonne provisoirement sa propre proposition 30J-32 J. Il ne la renie cependant pas, car le projet de Marc ne contredit pas le sien. Il l'intègre au contraire. Trois propriétés du couple $30 \mathrm{~J}-31 \mathrm{Ma}$ s'ajoutent pour le signaler. La première propriété est dialogique. L'enchaînement de $30 \mathrm{~J}$ à $31 \mathrm{Ma}$ s'effectue par une règle de dialogue de formation d'une disjonction dénommée par Carlson (1983) «D. or». (D. or) s'écrit ainsi : $<31 \mathrm{M} 1,\{30 \mathrm{~J}\},\{30 \mathrm{~J}\} \vdash 31 \mathrm{M} 1$, (D. or), Jeu de Question-Réponse $>$. «When a player has put forward a dialogue move, he may continue it by adjoining a further disjunct to it beginning with or » (Carlson, 1983 : 155). " one who adds a further disjunct to a move need not continue to endorse its premise. Neither does he have to accept the amendment. What he does have to accept is the completed disjunction, whether by accepting one of its disjuncts or by admitting several of them [...] By completing the first's speaker move, the second speaker here indicates he accepted it only as amended. Yet he need not consider the amendment any more likely than the other alternatives. » (Carlson, 1983, p. 159). En collaborant avec Marc, Jean n'abandonne pas sa propre option : il l'enrichit. La seconde propriété est discursive. $30 \mathrm{~J}$ et $31 \mathrm{M}$ sont deux questions propositionnelles. La première appelle une réponse en oui/non. La seconde élargit l'espace informationnel (Carlson, 1983), l'ensemble des options disponibles aux enfants à trois modes opératoires : la multiplication, la division et les deux. La troisième propriété est une propriété logique : un chemin logique relie la proposition que Jean soumet à l'approbation de Marc (30J) à la réponse que Jean fait implicitement à la question de Marc lorsqu'il adopte en actes la proposition de ce dernier (34J). Ecrivons combien a-t-il besoin d'étagères pour ranger ces cassettes? sous la forme du desideratum abstrait de cette question, soit : $\mathrm{C} / \mathrm{R}=\mathrm{E} / \mathrm{R} \times \mathrm{C} / \mathrm{E}$. Sachant qu'il y a 32 cassettes par étagère $(27 \mathrm{M}$, $29 \mathrm{M})$, il faut diviser $\mathrm{C} / \mathrm{R}$ par $32(30 \mathrm{~J})$ pour connaître le nombre d'étagères nécessaires au rangement $(\mathrm{E} / \mathrm{R})$. En effet :
1. $C / E=32$
2. $E / B=7$
Prémisse
3. $C / R=672$
Prémisse
4'. $C / R=E / R \times C / E$
5.. $C / R=E / R \times 32$
6'. $672=E / R \times 32$
Prémisse
Formule implicitement utilisée par Jean avançant sa proposition
Elim-1,4'
Elim-3,5' desideratum de la question de Jean 
Jean a certainement « en vue » une formule du genre de 6 ' quand il énonce $30 \mathrm{~J}$ et plus encore quand il énonce $32 \mathrm{~J}$. Mais ces énoncés sont trop inchoatifs pour être utiles dans l'interlocution. Jean ne semble apparemment pas avoir saisi ce que représente E/R. La proposition de Marc le sort de cette ornière ${ }^{(10)}$.

Alors que la première séquence permet de déterminer le nombre de cassettes contenu dans une bibliothèque, la seconde va utiliser ce résultat intermédiaire pour calculer la réponse au problème qui, satisfaisant le desideratum de la question de l'expérimentateur, clôt l'interaction, soit 9-13 dans la présentation axiomatique du raisonnement, soit encore $34 \mathrm{Ja}$-34Je pour la séquence correspondante. C'est Jean qui trouve le procédé qui conduira à la solution. Mais Marc l'adopte immédiatement après avoir refait le calcul de Jean $(35 \mathrm{Ma}-\mathrm{c})^{(11)}$. Le reste de la séquence sera ensuite consacré à la production de la solution et à sa présentation sur une feuille de papier, non sans quelques difficultés où l'ambiguïté du référent a encore sa part.

\section{Conclusion. Les enseignements de l'analyse interlocutoire de l'extrait}

L'intervalle conversationnel qui aura été mathématiquement fécond dans cette interlocution n'occupe finalement qu'un très bref instant, à peine quelques tours de parole sur les 72 que comporte la séquence. Mais ce sont des tours de parole très intriqués, produits par un couplage relationnel hautement interactif, fragile et, semble-t-il, plutôt non déterministe. Cela ne veut pas dire « irrationnel » pour autant. C'est plutôt que la productivité de l'interaction relève d'un faisceau causal où ce qui compte le plus c'est que des événements d'ordre différents (affectifs, cognitifs, sociaux, discursifs) concourent sans contradiction à l'étayage d'un raisonnement progressant vers la solution et situé, lui, au cœur du processus.

Existe-t-il un jeu de langage (comme on dit d'une fonction) qui interpolerait le processus logique mis en œuvre par les enfants dans leur délibération? Il y a en certainement plusieurs, mais celui qui nous semble se rapprocher le plus de l'extrait que nous avons analysé est le jeu de dialogue de recherche et de découverte. Ce jeu a été présenté par Hintikka dans plusieurs publications. L'exposé des bases épistémologiques de ces jeux et de ce qui les distingue des autres jeux, notamment de ce qui les distingue des jeux de dialogue logique (à la Lorenzen) nous entraînerait trop loin (mais voir Trognon \& Batt, 2010). On se contentera donc de souligner l'étroite ressemblance qui existe entre le vocabulaire que nous avons employé pour analyser la séquence et la description que Hintikka donne de ce jeu censé définir formellement ce que l'on entend par déduction ou

(10) Le raisonnement conduit par Jean en réponse à la question de Marc n'est pas en rupture avec le premier procédé qu'il propose. Nous montrons en effet dans d'autres publications qu'on peut glisser «naturellement» (et «formellement») de 30J à 34J1. Cependant, aborder ce point ici consommerait trop de signes. C'est pourquoi nous nous permettrons de renvoyer à Trognon et Batt (2011).

(11) Notez que Marc ne se trompe pas de référent. $C f .35 \mathrm{Ma}$ : par étagère... 32. 
par inférence dans le discours ordinaire, où l'on combine dans un raisonnement des prémisses et des réponses à des questions judicieusement choisies ( $C f$. Trognon, Batt \& Saint-Dizier, 2010). Les règles proposées par Hintikka décrivent des mouvements qui sont ceux-là même que nous avons évoqués : mouvements interrogatif, définitionnel, assertorique et déductif, eux-mêmes expliqués en termes de séquents. Le jeu de dialogue de recherche et de découverte constitue ainsi selon nous un bon modèle du processus psycho-socio-discursif qui a permis aux enfants de résoudre le problème. Il suffit d'ajouter à ce modèle le schéma de l'apprentissage dans l'interaction (Trognon \& Batt, 2003, 2010) pour qu'il devienne un élément d'une explication des bénéfices cognitifs de l'interaction. En effet, nous l'avons déjà souligné plus haut, 34J est l'un des deux moments clés du processus de découverte de la solution, sinon le plus important. Qu'est-ce que Jean fait à moment-là ? Il effectue dans une sorte de monologue intérieur Vygotskien (Wetsch, 1979) mi-dans mi-hors interaction, à la fois « dans sa tête » et " dans l'interaction », un calcul (mouvement déductif du jeu de dialogue de recherche et de découverte) ayant parmi ses données le désideratum de la question de Marc (mouvement interrogatif du jeu de dialogue de recherche et de découverte), puis il attribue une définition au résultat de ce calcul (mouvement définitionnel du jeu de dialogue de recherche et de découverte). Cette succession de mouvements conversationnel consiste à élaborer un raisonnement qui prend pour prémisse une idée de (demandée par) Marc pour l'intégrer à des connaissances propres. C'est très exactement la formule de l'apprentissage dans l'interaction (Trognon et Batt, 2003, 2010).

Il reste à nous demander si le contexte créé par l'interlocution aura permis aux enfants de montrer aux observateurs que nous sommes leur connaissance de la division. Si nous ne doutons pas que l'interaction leur a fait expériementer des aspects importants de la division, nous sommes bien loin d'être assurés que c'est le concept de division qu'ils ont accompli au cours de leur jeu de langage. Le procédé trouvé par les enfants peut se décrire grâce à la formule de la division: $\mathrm{a}=\mathrm{bc}+\mathrm{q}$, que nous réécrivons :

$$
\begin{aligned}
& 672=224 \times 3+0, \text { puis } \\
& 672-224 \times 3=0 \\
& 672-224-224-224=0
\end{aligned}
$$

La troisième ligne ci-dessus pourrait s'interpréter comme un « théorème en actes » selon Vergnaud, en l'occurrence une "division en actes ». Mais d'un point de vue conceptuel, s'agit-il bien d'une division? Certes, le procédé se perçoit bien au final comme l'effectuation d'une division. Mais si on appelle « division » le fait de construire l'idée qu'un ensemble peut se découper virtuellement en parts égales, en quelque sorte avant de le faire réellement, ce que les enfants ont réalisé n'est pas à proprement parler une division, mais plutôt une somme de soustractions. En enlevant 224 cassettes du tas de cassettes, il n'en reste plus que 448 à ranger (ligne 1 ci-dessous). Or enlever 224 cassettes du tas, c'est remplir donc utiliser une bibliothèque (ligne 2). On fait la même opération une seconde fois (lignes 3-4) et il reste encore 224 cassettes à « caser». On fait la même opération une troisième fois (lignes 5-6) et il ne reste plus de cassettes à ranger, le tas est épuisé. On aura donc utilisé 3 bibliothèques en tout pour ranger les cassettes. 
En effet, Jean propose en fait l'opération :

1. $672-224=448$

2. $672-\mathrm{C} / \mathrm{B}=448$

3. $672-\mathrm{C} / \mathrm{B}=224=224$

4. $672-\mathrm{C} / \mathrm{B}-\mathrm{C} / \mathrm{B}=224$

5. $672-\mathrm{C} / \mathrm{B}-\mathrm{C} / \mathrm{B}-224=0$

6. $672-\mathrm{C} / \mathrm{B}-\mathrm{C} / \mathrm{B}-\mathrm{C} / \mathrm{B}=0$

7. $672-3 \times \mathrm{C} / \mathrm{B}=0$

8. $672=3 \times \mathrm{C} / \mathrm{B}$

Il se pourrait donc que les enfants aient trouvé un procédé aboutissant au même résultat qu'une division tout en n'ayant pas fait (ou eu à faire) une division. D'une certaine façon, ce serait un abus de langage de leur attribuer à l'issue de cette interaction la connaissance de la division. En effet, pour résoudre le problème qui leur est posé, les enfants ont recours à un procédé moins élaboré cognitivement que celui qui est visé par le problème. Pourquoi donc les enfants se «casseraient-ils la tête » pour résoudre un problème qu'ils savent solutionner plus simplement? Si notre hypothèse est juste, l'interaction aura mobilisé une heuristique plutôt qu'un algorithme. Il aurait fallu un problème ne pouvant être solutionné qu'avec des moyens nouveaux pour prouver l'efficacité de l'interaction dans l'acquisition de compétences nouvelles. Mais disons que l'interaction présentée ici ne permet pas de trancher sur la possession par les enfants de cette compétence. Reste qu'en obtenant le résultat d'une division sans faire exactement une division, les enfants obéissent à la consigne, puisque celle-ci leur demandait de faire des multiplications. Les enfants ne sont certainement pas conscients de cette assez extraordinaire conséquence, où il faut voir la main de Dame Nature, mais elle vaut d'être soulignée ! 


\section{Références}

BARTH, E. M. \& KRABBE, E. C. W. (1982) : From axiom to dialogue, Berlin, N. Y. : Walter de Gruyter.

CARlson, L. (1983) : Dialogue games. An approach to discourse Analysis, Dordrecht : Reidel.

CARON, Jean. (1997) : «Psychologie cognitive et interactions conversationnelles », in Conversation, interaction et fonctionnement cognitif, Josie Bernicot, Alain Trognon and Josiane Caron-Pargue (eds), 221-238. Nancy : Presses Universitaires de Nancy.

DaVid, R., Nour, K. \& RAfFalli, C. (2001) : Introduction à la logique, Paris : Dunod.

GARfinkel, H. (1990) : «The Curious Seriousness of Professional Sociology », in Les formes de la conversation, Bernard Conein, Michel de Fornel and Louis Quéré (eds.), 1, 145-161. Paris : CNET.

Gilly, M., RouX, J.-P., \& Trognon, A. (1999) : Apprendre dans l'interaction, Nancy : Presses Universitaires de Nancy, Aix-en-Provence : Publications de l'université de Provence.

HintikKA, J. (1981) : «Questions de réponses et bien d'autres questions encore », Langue Française, 52, 56-69.

- (2007): Les principes des mathématiques revisitées, Paris : Vrin (The Principles of Mathematics Revisited, Cambrige University Press, 1996)

INHELDER, B. \& DE CAPRONA, D. (1992) : «Vers le constructivisme psychologique : Structures ? Procédures ? Les deux indissociables ", in B. Inhelder, .G. Cellérier, E. Ackermann, A.Blanchet, D. De Caprona, J.J. Ducret, \& M. Saada-Robert, (Eds.). Le cheminement des découvertes de l'enfant (pp. 19-50). Neuchâtel : Delachaux \& Niestlé.

PERRET-Clermont, A. N. (1979) : La construction de l'intelligence dans l'interaction sociale, Peter Lang, Berne.

POLITZER, J. (1928/1968) : Critique des fondements de la psychologie, Paris : PUF.

REITER, R. (1980) : «A logic for default reasoning », Artificial Intelligence 13 : 81 132.

SÄLJÖ, R., \& WYNDHAMN, J. (1990) : «Problem-solving, academic performance and situated reasoning a study of joint cognitive activity in formal setting », British Journal of Educational Psychology, 60, 245-254.

Schegloff, E.A. (199) : "Conversation Analysis and Socially Shared Cognition ", in Perspectives on socially shared cognition, Lauren B. Resnick, John M. Levine and Stephanie D. Teasley (eds.), 150-170. Washington : American Psychological Association, DC.

Sorsana, C. (1999) : Psychologie des interactions sociocognitives, Paris : Armand Colin.

TrognOn, A. (2002) : "Speech Acts and the Logic of Mutual Understanding », in Essays in Speech Acts Theory, Daniel Vanderveken, Susumo Kubo (eds), 121 133. Amsterdam : John Benjamins and sons.

Trognon, A., \& BATT, M. (2003) : « Comment représenter le passage de l'Intersubjectif à l'Intrasubjectif? Essai de Logique Interlocutoire », L'Orientation Scolaire et Professionnelle, 32, 3, 399-436. 
— (2010) : «Interlocutory Logic : A Unified Framework for Studying Conversation Interaction », in J. Streek (ed.), New Adventures in Language and Interaction, pp. 9-40. Bruxelles : Benjamins.

- (2010): «Some Aspects of the Current Situation in Research on Dialogue Logic : Introduction to the Logical Properties of Dialogue ", in A. Trognon, M. Batt, J. Caelen et D. Vernant (eds.), Logical Properties of Dialogue. Nancy: Presses universitaires de Nancy.

Trognon, A., Batt, M. \& SAINT DizIER, V. (2010) : «Dialogue games of argumentation », in Dialogue's logical properties, Alain Trognon, Martine Batt, Jean Caelen and Denis Vernant. Nancy : Presses Universitaires de Nancy.

Trognon, A. \& Bromberg, M. (2007) : «L'interaction sociale », in Psychologie sociale et ressources humaines, Alain Trognon and Marcel Bromberg (eds.), 63-94. Paris : PUF.

Trognon, A. \& Coulon, D. (2001) : «La modélisation des raisonnements générés dans 1'interlocution », Langages, 144, 58-77.

Trognon, A., SAint-Dizier de AlmeidA, V., \& Grossen, M. (1999) : « Résolution conjointe d'un problème arithmétique », in M. Gilly, J.-P. Roux \& A. Trognon (Eds.), Apprendre dans l'interaction. Analyse des médiations sémiotiques. Aix-en-Provence \& Nancy : Publications de 1'Université de Provence $\&$ Presses Universitaires de Nancy.

Trognon, A., Sorsana, C. (2005) : «Les compétences interactionnelles : formes d'exercice, bases, effets et développement », Rééducation Orthophonique, 221, Avril 2005, 29-56.

Vygotski, L. S. (1934) : Myshlenie i rech' : Psikhologicheskie issledovaniya. Moscow and Leningrad : Gosudarstvennoe Sotsial'no-Ekonomicheskoe Isdatel'stvo.

W Alton, D. (2007) : Dialog Theory for Critical Argumentation, Amsterdam : John Benjamins Publishing Company

WeRTSCH, J. (1979) : «From Social Interaction to Higher Psychological Processes. A clarification and Application of Vygotsky's Theory ", Human Development, 1979, 22, 1-22.

WERTSCH, J. S AMMARCO, J. (1988) : «Précurseurs sociaux du fonctionnement cognitif individuel : le problème des unités d'analyse », in R. Hinde, A. N. PerretClermont \& J. Stevenson-Hinde (eds.), Relations interpersonnelles et développement des savoirs (pp. 395-415). Berne : Delval. 